\title{
Complete sets of invariants for dynamical systems that admit a separation of variables
}

\author{
E. G. Kalnins ${ }^{\text {a) }}$ and J. M. Kress ${ }^{\text {b) }}$ \\ Department of Mathematics, University of Waikato, Hamilton, New Zealand \\ W. Miller, Jr. ${ }^{\mathrm{c})}$ \\ School of Mathematics, University of Minnesota, Minneapolis, Minnesota, 55455 \\ G. S. Pogosyan ${ }^{\text {d) }}$ \\ Centro de Ciencias Físicas, Universidad Nacional Autónoma de México, \\ Apartado Postal 48-3, 62251 Cuernavaca, Morelos, México
}

(Received 7 March 2002; accepted for publication 20 March 2002)

Consider a classical Hamiltonian $H$ in $n$ dimensions consisting of a kinetic energy term plus a potential. If the associated Hamilton-Jacobi equation admits an orthogonal separation of variables, then it is possible to generate algorithmically a canonical basis $\mathbf{Q}, \mathbf{P}$ where $P_{1}=H, P_{2}, \ldots, P_{n}$ are the other second-order constants of the motion associated with the separable coordinates, and $\left\{Q_{i}, Q_{j}\right\}=\left\{P_{i}, P_{j}\right\}$ $=0,\left\{Q_{i}, P_{j}\right\}=\delta_{i j}$. The $2 n-1$ functions $Q_{2}, \ldots, Q_{n}, P_{1}, \ldots, P_{n}$ form a basis for the invariants. We show how to determine for exactly which spaces and potentials the invariant $Q_{j}$ is a polynomial in the original momenta. We shed light on the general question of exactly when the Hamiltonian admits a constant of the motion that is polynomial in the momenta. For $n=2$ we go further and consider all cases where the Hamilton-Jacobi equation admits a second-order constant of the motion, not necessarily associated with orthogonal separable coordinates, or even separable coordinates at all. In each of these cases we construct an additional constant of the motion. (C) 2002 American Institute of Physics. [DOI: 10.1063/1.1484540]

\section{INTRODUCTION}

The quest for integrable systems has a long history. Basically, the question is, given a classical Hamiltonian $H=H(x, p)$ where $\mathbf{x}=\left(x_{1}, \ldots, x_{n}\right), \mathbf{p}=\left(p_{1}, \ldots, p_{n}\right)$, how can one find all the solutions to the Poisson bracket condition

$$
\{H, L\}=\sum_{i=1}^{n}\left(\frac{\partial H}{\partial p_{i}} \frac{\partial L}{\partial x_{i}}-\frac{\partial H}{\partial x_{i}} \frac{\partial L}{\partial p_{i}}\right)=0
$$

where $L=L(\mathbf{x}, \mathbf{p}) .^{1}$ There is no known comprehensive solution to this problem. However, if the associated Hamilton-Jacobi equation $H(\mathbf{x}, \partial S / \partial \mathbf{x})=E$ is additively separable in the orthogonal variables $\mathbf{x}$, then a complete integral of the equation can be constructed by quadratures and one can find a basis of $2 n-1$ functionally independent solutions to Eq. (1). Indeed there is an explicit canonical change of coordinates from the variables $\mathbf{x}, \mathbf{p}$ with $\left\{x_{i}, p_{j}\right\}=\delta_{i j}$ to variables $\mathbf{Q}, \mathbf{P}$ where $P_{1}=H, P_{2}, \ldots, P_{n}$ are the other second-order constants of the motion associated with the orthogo-

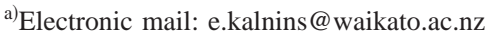

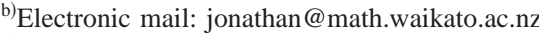

${ }^{c}$ Electronic mail: miller@ima.umn.edu

${ }^{d)}$ Permanent address: Laboratory of Theoretical Physics, Joint Institute for Nuclear Research, Dubna, Moscow Region, 141980, Russia and International Center for Advanced Studies, Yerevan State University, A. Manougian 1, 375049, Yerevan, Armenia.
} 
nal separable $x$-coordinates, and $\left\{Q_{i}, Q_{j}\right\}=\left\{P_{i}, P_{j}\right\}=0,\left\{Q_{i}, P_{j}\right\}=\delta_{i j}$. Thus the $2 n-1$ functions $Q_{2}, \ldots, Q_{n}, P_{1}, \ldots, P_{n}$ form a basis for the invariants. Each invariant $Q_{j}$ can be expressed as a sum of the form

$$
Q_{j}=\sum_{k=1}^{n} M_{k}\left(x_{k}, \mathbf{P}\right)
$$

see Ref. 1.

Numerous examples have been found through this approach, but important problems remain. Many of the known interesting dynamical systems have extra constants of the motion $L$ which are polynomial in the canonical momenta $p_{i}, i=1, \ldots, n$. This often enables global statements to be made about the system in question, e.g., the existence of closed orbits. However, though many interesting results have been obtained, e.g., Refs. 2 and 3, an algorithmic way of generating all polynomial solutions to (1) is not known. In particular, from the $x$-based integrals in (2) it is difficult to tell if $Q_{j}$ is a polynomial in the momenta $p_{i}$. In this article we adopt a $p$-based approach to the calculation of the invariants $Q_{j}$ in which the term $M_{k}$ take the form $M_{k}$ $=M\left(p_{k}, \mathbf{P}\right)$, and we can say in advance for exactly which separable metrics and potentials $Q_{j}$ is a polynomial in the momenta. We give, in principle, a complete solution to this problem. Moreover, we show how to characterize each term $M_{k}$ in (2) by the Poisson brackets $\left\{M_{k}, P_{j}\right\}$. [Note: Although the term $M_{k}\left(x_{k}, \mathbf{P}\right)$ always exists, there are cases where it cannot be expressed as $M_{k}\left(p_{k}, \mathbf{P}\right)$, i.e., as a function of $p_{k}$ alone. These are exactly the cases where $x_{k}$ is an ignorable variable, i.e., where the components of the metric tensor in the $\mathbf{x}$-coordinates do not depend on $x_{k}$ and where, also, the potential $V$ does not depend on $x_{k}$. However, these special cases where $M_{k}$ and the invariant $Q_{i}$ of which it is a component term always have polynomial dependence (after multiplication by a linear combination of second-order invariants) can be handled separately or by requiring that $M_{k}$ depends on a variable with some $x$ dependence, such as $M_{k}\left(r\left(x_{k}\right) p_{k}, \mathbf{P}\right)$ treated below.]

Of course, the system could admit a polynomial invariant

$$
L=R\left(\mathbf{P}, Q_{2}, \ldots, Q_{n}\right)
$$

such that $L, \mathbf{P}$ is functionally independent, even if $Q_{2}, \ldots, Q_{n}$ are not polynomials. It is a much more difficult problem to classify all such possibilities for polynomial $L$ as functions of possibly nonpolynomial $Q_{j}$. We make some progress toward the solution of this problem, through the consideration of important examples. These questions of when a system with $n$ second-order constants of the motion (generated by an orthogonal separation of variables) admits additional polynomial constants of the motion are closely related to the concept of superintegrability. ${ }^{4-18}$

For dimension $n=2$ in this article, we go beyond the formulation discussed above and consider all cases where the Hamilton-Jacobi equation admits a second-order constant of the motion, not necessarily associated with orthogonal separable coordinates, or even separable coordinates at all. In each of these cases we construct an additional constant of the motion.

\section{CARTESIAN SYSTEMS IN TWO DIMENSIONS}

Let us first consider two dimensional Euclidean space. In Cartesian coordinates the Hamiltonian $H$ has the form

$$
H=p_{x}^{2}+p_{y}^{2}+V(x, y) .
$$

If we have separation of variables in Cartesian coordinates the potential must take the form

$$
V(x, y)=X(x)+Y(y) .
$$


We immediately observe that there are already two invariants arising from the separation, namely $L_{1}=p_{x}^{2}+X(x)$ and $L_{2}=p_{y}^{2}+Y(y)$. Our problem is to calculate a third invariant and determine when it can be chosen to be a polynomial in the canonical momenta. To do this we compute two functions $M\left(x, p_{x}\right)$ and $N\left(y, p_{y}\right)$ that satisfy the conditions

$$
\{H, M\}=1, \quad\{H, N\}=1 .
$$

These equations can be solved in principle if we know the original functions $X$ and $Y$. Indeed, if we write out the first of these conditions, we obtain

$$
2 p_{x} \frac{\partial M}{\partial x}-X^{\prime} \frac{\partial M}{\partial p_{x}}=1
$$

This equation can be readily solved to give

$$
M=-\int X^{\prime-1} d Q
$$

where $Q=p_{x}$ and $L_{1}=p_{x}^{2}+X$. [We consider $X^{\prime-1}=d x / d X$ to be a function of $X=L_{1}-Q^{2}$ to compute the integral. An arbitrary function $f\left(L_{1}, L_{2}\right)$ can be added to the integral, but this makes no difference since $L_{1}, L_{2}$ are invariants.] Once $M$ and $N$ have been determined, we see that $L_{3}$ $=N-M$ must be an invariant. It is immediately clear that if $X=x^{1 / p}$ where $p$ is an integer, then $M$ is a polynomial in $p_{x}$. As examples of this consider the following.

(1) $p=3$ :

$$
M=-3 x^{2 / 3} p_{x}-4 x^{1 / 3} p_{x}^{3}-\frac{8}{5} p_{x}^{5}
$$

(2) $p=4$ :

$$
M=-4 x^{3 / 4} p_{x}-8 x^{1 / 2} p_{x}^{3}-\frac{32}{5} x^{1 / 4} p_{x}^{5}-\frac{64}{35} p_{x}^{7} .
$$

It follows from these two examples that the Hamiltonian

$$
H=p_{x}^{2}+p_{y}^{2}+x^{1 / 3}+y^{1 / 4}
$$

has, in addition to the obvious invariants

$$
L_{1}=p_{x}^{2}+x^{1 / 3}, \quad L_{2}=p_{y}^{2}+y^{1 / 4},
$$

the additional invariant

$$
L_{3}=3 x^{2 / 3} p_{x}+4 x^{1 / 3} p_{x}^{3}+\frac{8}{5} p_{x}^{5}-4 y^{3 / 4} p_{y}-8 y^{1 / 2} p_{y}^{3}-\frac{32}{5} y^{1 / 4} p_{y}^{5}-\frac{64}{35} p_{y}^{7} .
$$

From this observation we conclude that all potentials of the form

$$
V=\alpha x^{1 / p}+\beta y^{1 / q}
$$

have the superintegrability property with three functionally independent invariants which are polynomial in $p_{x}$ and $p_{y}$. This includes the known examples corresponding to $p=1,2$. If $X(x)$ is determined by a polynomial relation of the form

$$
\sum_{j=1}^{n} a_{j} X^{j}=x
$$


we can go even further. Then the function $M$ is always a polynomial in the canonical momentum $p_{x}$. As an example consider

$$
X(x)=2^{-1 / 3}\left[\left\{x+\sqrt{x^{2}+1}\right\}^{1 / 3}-\left\{x+\sqrt{x^{2}+1}\right\}^{-1 / 3}\right] .
$$

The inverse function is

$$
x=X^{3}+\frac{3}{2^{2 / 3}} X
$$

and the corresponding function $M\left(x, p_{x}\right)$ is given by

$$
-M\left(x, p_{x}\right)=\frac{8}{5} p_{x}^{5}+4 X p_{x}^{3}+3 X^{2} p_{x}+\frac{3}{2^{2 / 3}} p_{x} .
$$

It is clear that all that we have done applies also to potentials that separate in $n$ dimensions, in Cartesian coordinates. There is only one further Cartesian case for which polynomial invariants can be generated. Let us consider the case when $X(x)=\omega_{1}^{2} x^{2}$. The corresponding function $M\left(x, p_{x}\right)$ is given by

$$
M\left(x, p_{x}\right)=\frac{1}{4 \omega_{1}} \arcsin \left(\frac{\omega_{1}^{2} x^{2}-p_{x}^{2}}{\omega_{1}^{2} x^{2}+p_{x}^{2}}\right) .
$$

If $Y(y)=\omega_{2}^{2} y^{2}$, this establishes that the Hamiltonian

$$
H=p_{x}^{2}+p_{y}^{2}+\omega_{1}^{2} x^{2}+\omega_{2}^{2} y^{2}
$$

has the constant of motion

$$
L_{3}=\frac{1}{4 \omega_{1}} \arcsin \left(\frac{\omega_{1}^{2} x^{2}-p_{x}^{2}}{\omega_{1}^{2} x^{2}+p_{x}^{2}}\right)-\frac{1}{4 \omega_{2}} \arcsin \left(\frac{\omega_{2}^{2} y^{2}-p_{y}^{2}}{\omega_{2}^{2} y^{2}+p_{y}^{2}}\right),
$$

in addition to the constants $L_{1}=p_{x}^{2}+\omega_{1}^{2} x^{2}$ and $L_{2}=p_{y}^{2}+\omega_{2}^{2} y^{2}$. In general this invariant is not polynomial in the canonical momenta. However, if $\omega_{1} / \omega_{2}$ is a fraction $p / q$ for integers $p, q$, then $\omega_{1}=p s, \omega_{2}=q s$ and $L_{3}^{\prime}=\sin \left(4 s p q L_{3}\right)$ will be a rational invariant whose common denominator is a product of powers of $L_{1}$ and $L_{2}$. The numerator is then an additional polynomial invariant, e.g., consider $\omega_{1}=1, \omega_{2}=2$. Then

$$
L_{3}^{\prime}=\sin \left(8 L_{3}\right)=\frac{L_{1} L_{2}^{2}-2\left(x p_{y}^{2}-4 y p_{x} p_{y}-4 x y^{2}\right)^{2}}{L_{1} L_{2}^{2}},
$$

which indicates that $L_{3}^{\prime \prime}=x p_{y}^{2}-4 y p_{x} p_{y}-4 x y^{2}$ is an additional invariant. In general, $L_{1}^{p} L_{2}^{q} \sin \left(4 s p q L_{3}\right)$ will be a polynomial invariant, functionally independent of $L_{1}$ and $L_{2}$.

\section{GENERAL TWO-DIMENSIONAL SEPARABLE SYSTEMS}

If we extend this problem to the case of orthogonal separable coordinates in a general Riemannian space, we know that the Hamiltonian in a given set of coordinates with a separable potential has the form

$$
H=L_{1}=\frac{p_{x}^{2}+p_{y}^{2}+v_{1}(x)+v_{2}(y)}{f_{1}(x)+f_{2}(y)},
$$

and, due to the separability, there is the invariant ${ }^{19-21}$ 


$$
L_{2}=\frac{f_{2}(y)\left(p_{x}^{2}+v_{1}(x)\right)-f_{1}(x)\left(p_{y}^{2}+v_{2}(y)\right)}{f_{1}(x)+f_{2}(y)} .
$$

We can implement the same ansatz as we have done previously by looking for a function $M\left(H, x, p_{x}\right)$ which satisfies

$$
\{H, M\}=\frac{1}{f_{1}(x)+f_{2}(y)} .
$$

The condition has the form

$$
\left(-v_{1}^{\prime}(x)+f_{1}^{\prime}(x) H\right) \frac{\partial M}{\partial p_{x}}+2 p_{x} \frac{\partial M}{\partial x}=1 .
$$

Assuming that $\left|v_{1}^{\prime}\right|+\left|f_{1}^{\prime}\right|>0$, we see that this equation has the solution

$$
M\left(H, L_{2}, p_{x}\right)=\int U^{\prime-1} d Q
$$

where

$$
Q=p_{x}, \quad L_{2}=v_{1}(x)-f_{1}(x) H+p_{x}^{2}, \quad U(x)=-v_{1}(x)+f_{1}(x) H+L_{2} .
$$

[We consider $U^{\prime-1}=d x / d U$ to be a function of $U=Q^{2}$. An arbitrary function $f\left(L_{1}, L_{2}\right)$ can be added to the integral, but this makes no difference since $L_{1}=H$ and $L_{2}$ are invariants.] There is a similar condition for the function $N\left(H, L_{2}, y, p_{y}\right)$. The new invariant is $L_{3}=N-M$. It is straightforward to verify the condition

$$
\left\{L_{2}, L_{3}\right\}=1 \text {. }
$$

Indeed, $\left\{L_{2}, M\right\}=f_{2} /\left(f_{1}+f_{2}\right),\left\{L_{2}, N\right\}=-f_{1} /\left(f_{1}+f_{2}\right)$. This implies that the set $L_{1}, L_{2}, L_{3}$ is functionally independent.

Similarly, we can construct functions $M\left(H, x, p_{x}\right), N\left(H, y, p_{y}\right)$ that satisfy

$$
\{H, M\}=\frac{f_{1}(x)}{f_{1}(x)+f_{2}(y)}, \quad\{H, N\}=\frac{-f_{2}(y)}{f_{1}(x)+f_{2}(y)} .
$$

Assuming that $\left|v_{i}^{\prime}\right|+\left|f_{i}^{\prime}\right|>0$ for $i=1,2$, we see that these equations have the solutions

$$
M\left(H, L_{2}, p_{x}\right)=\int f_{1}(x) U_{1}^{\prime-1} d Q, \quad N\left(H, L_{2}, p_{y}\right)=-\int f_{2}(y) U_{2}^{\prime-1} d Q,
$$

where

$$
U_{i}=-v_{i}+f_{i} H+L_{2} .
$$

Setting $L_{4}=N-M$, we see that $L_{4}$, not an invariant, satisfies

$$
\left\{H, L_{4}\right\}=1, \quad\left\{L_{2}, L_{4}\right\}=0 .
$$

Let us illustrate what can happen with some examples.

(1) We choose parabolic coordinates in Euclidean space ${ }^{22} x^{\prime}=1 / 2\left(\xi^{2}-\eta^{2}\right), y^{\prime}=\xi \eta$. First consider the parabolic-separable Hamiltonian 


$$
H=L_{1}=\frac{p_{\xi}^{2}+p_{\eta}^{2}+\xi}{\xi^{2}+\eta^{2}}
$$

We can immediately associate with this the extra invariant

$$
L_{2}=\frac{\eta^{2} p_{\xi}^{2}-\xi^{2} p_{\eta}^{2}+\eta^{2} \xi}{\xi^{2}+\eta^{2}}
$$

If we look for our functions $M\left(\xi, p_{\xi}\right)$ and $N\left(\eta, p_{\eta}\right)$, as before we obtain

$$
\begin{gathered}
M\left(\xi, p_{\xi}\right)=\frac{1}{4 \sqrt{H}} \ln \left(\begin{array}{c}
\sqrt{H} p_{\xi}+\frac{1}{2}-\xi H \\
-\sqrt{H} p_{\xi}+\frac{1}{2}-\xi H
\end{array}\right), \\
N\left(\eta, p_{\eta}\right)=\frac{1}{4 \sqrt{H}} \ln \left(\frac{\sqrt{H} \eta+p_{\eta}}{\sqrt{H} \eta-p_{\eta}}\right) .
\end{gathered}
$$

If we now consider the constant $\cosh (4(M-N) \sqrt{H})$, we find that it can be written in the form

$$
4 \cosh (4(M-N) \sqrt{H})=\frac{L_{3}^{2} H}{\left(1-4 H L_{2}\right) L_{2}},
$$

where

$$
L_{3}=\frac{2 \xi \eta}{\xi^{2}+\eta^{2}}\left(p_{\xi}^{2}+p_{\eta}^{2}\right)-2 p_{\xi} p_{\eta}+\frac{\eta\left(\xi^{2}-\eta^{2}\right)}{\xi^{2}+\eta^{2}}
$$

is an additional invariant quadratic in the canonical momenta. This is a special case of a more general example in Ref. 23.

(2) Consider the Hamiltonian in Cartesian coordinates

$$
H=p_{x}^{2}+p_{y}^{2}+\frac{x}{\sqrt{x^{2}+y^{2}}}
$$

In parabolic coordinates this Hamiltonian has the form

$$
H=L_{1}=\frac{p_{\xi}^{2}+p_{\eta}^{2}+\xi^{2}-\eta^{2}}{\xi^{2}+\eta^{2}} .
$$

The second-order invariant associated with this separation is

$$
L_{2}=\frac{\xi^{2} p_{\eta}^{2}-\eta^{2} p_{\xi}^{2}-2 \xi^{2} \eta^{2}}{\xi^{2}+\eta^{2}} .
$$

The additional invariant calculated by our method is given by

$$
\begin{aligned}
L_{3}= & \frac{\operatorname{arccosh}\left(\left[(H-1) \xi^{2}+p_{\xi}^{2}\right] /\left[(H-1) \xi^{2}-p_{\xi}^{2}\right]\right)}{\sqrt{H-1}} \\
& +\frac{\operatorname{arccosh}\left(\left[(H+1) \eta^{2}+p_{\eta}^{2}\right] /\left[(H+1) \eta^{2}-p_{\eta}^{2}\right]\right)}{\sqrt{H+1}}
\end{aligned}
$$


which is clearly transcendental.

(3) If we consider the Hamiltonian

$$
H=p_{x}^{2}+p_{y}^{2}+i b(x+i y),
$$

then using the semihyperbolic coordinates ${ }^{22}$

$$
x+i y=i(u+w), \quad x-i y=(-i / 2)(u-w)^{2}
$$

and applying our construction, we find

$$
\frac{\exp (M-N)-i}{\exp (M-N)+i}=-i \frac{\sqrt{\bar{b}-i X}}{\sqrt{b+i X}},
$$

thus giving rise to the additional constant $X=p_{x}+i p_{y}$.

(4) Let us now look at an example of a potential where our construction yields elliptic integrals. We consider the potential $V=2 x+\beta / y^{2}$. If we carry out the construction using parabolic coordinates $x=\left(\frac{1}{2}\right)\left(\xi^{2}-\eta^{2}\right), y=\xi \eta$, then the functions $M$ and $N$ are given by the integrals

$$
M=\frac{1}{2} \int \frac{\xi d \xi}{\sqrt{-\xi^{6}+H \xi^{4}+L \xi^{2}-\beta}}, \quad N=\frac{1}{2} \int \frac{\eta d \eta}{\sqrt{\eta^{6}+H \eta^{4}+L \eta^{2}-\beta}},
$$

where $L$ is the quadratic constant associated with the separation of variables in parabolic coordinates. If we change variables according to $u=\xi^{2}, v=-\eta^{2}$, then both $M$ and $N$ are given by integrals of the form

$$
I=\frac{1}{2} \int \frac{d \lambda}{\sqrt{(a-\lambda)(b-\lambda)(c-\lambda)}},
$$

where $\lambda=u, v$ and

$$
a b c=-\beta, \quad L=a b+b c+a c, \quad H=a+b+c .
$$

There are a variety of ways of evaluating elliptic integrals of this type. We recall that all our considerations are in the complex domain. As an example, we can choose to use the complex equivalent of the integral

$$
\int_{-\infty}^{u} \frac{d x}{\sqrt{(a-x)(b-x)(c-x)}}=\frac{2}{\sqrt{a-c}} F(\alpha, p),
$$

valid for $a>b>c \geqslant u$ and for which

$$
\sqrt{\frac{a-c}{a-u}}=\sin \alpha=\operatorname{sn}(A, p), \quad p=\sqrt{\frac{a-b}{a-c}} .
$$

Then if we calculate $\operatorname{sn}^{2}(\sqrt{a-c}(M-N), p)$ using the addition formulas for elliptic functions we obtain

$$
\operatorname{sn}^{2}(\sqrt{a-c}(M-N), p)=\frac{c-a}{c+b+L_{1}},
$$


where $L_{1}$ is the second quadratic constant associated with this super-integrable system. Because of the various ways of evaluating elliptic integrals, there are a number of ways of uncovering the presence of $L_{1}$.

In analogy with the constructions (5)-(7), we can find Riemannian spaces and potentials with polynomial invariants of arbitrarily high order. Set

$$
x=P_{n}\left(\frac{U+A}{\alpha+\beta H}\right), \quad A=\delta+\phi H-L_{2},
$$

where $P_{n}$ is a polynomial of order $n$ and $\alpha, \beta, \delta, \phi$ are constants. Then there exists a function $F_{n}$, inverse to $P_{n}$, i.e., $F_{n}\left(P_{n}(y)\right)=y$, such that

$$
U=(\alpha+\beta H) F_{n}(x)-\delta-\phi H+L_{2},
$$

and $v_{1}(x)=-\alpha F_{n}(x)+\delta, f_{1}(x)=\beta F_{n}(x)-\phi$, where $(\alpha+\beta H)^{n} M\left(x, p_{x}\right)$ is a polynomial in the momenta. The Cartesian coordinate constructions (5)-(7) correspond to the special case $\beta=0$.

The solution of the equation (11) can be understood in a more general context. We have the dual relations

$$
x=F\left(U-L_{2}, H\right), \quad U(x, H)=-v_{1}(x)+f_{1}(x) H+L_{2}, \quad U_{x} \neq 0 .
$$

(Since $U$ and $L_{2}$ occur only as $U-L_{2}$, we will, without loss of generality, set $L_{2}=0$ in the theoretical developments to follow, and then replace $U$ by $U-L_{2}$ in the examples.) Thus we have

$$
1=F_{U} U_{x}, \quad F_{U} U_{H}+F_{H}=0 .
$$

The condition that $U(x, H)$ is linear in $H$, i.e., $U_{H H}=0$, leads to the following necessary and sufficient conditions that the function $x=F(U, H)$ correspond to an invariant $M$ on a Riemannian manifold with potential:

$$
F_{H H} F_{U}^{2}-2 F_{U H} F_{U} F_{H}+F_{U U} F_{H}^{2}=0, \quad F_{U} \neq 0 .
$$

This equation admits an infinite dimensional conformal symmetry group. Indeed if $V=F(U, H)$ is a solution, then $G(V)$ is also a solution, for any nonconstant function $G$. Also, this group contains the subgroup of inhomogeneous affine symmetries: if $F(U, H)$ is a solution, then so is $F\left(\left[a_{11} U\right.\right.$ $\left.+a_{12} H++a_{13}\right] / A,\left[a_{21} U+a_{22} H+a_{23}+a_{23}\right] / A$ ), where $a_{i j}$ are constants, $\operatorname{det}\left(a_{i j}\right) \neq 0$ and

$$
A=a_{31} U+a_{32} H++a_{33} .
$$

Note that the function $V_{1}=(U+\delta+\phi H) /(\alpha+\beta H)$ satisfies (23), so any function of $V_{1}$ must also satisfy the requirement. This puts (21) in the proper context. A more general solution is $V_{2}$ $=(U+\phi H+\delta) /(\alpha U+\beta H+\gamma)$, where again any function of $V_{2}$ also satisfies the requirement. Equation (23) also occurs in the theory of level sets, used in computational geometry and computer vision, ${ }^{24}$ since it describes the family of functions $F$ whose level sets are always straight lines in the $(U, H)$ plane.

We have seen that the construction (21) always leads to a polynomial invariant $L_{3}$, up to multiplication by a polynomial in $H$ and $L_{2}$. In fact these are the only polynomial invariants $L_{3}$ that can be constructed directly from the integration. This follows from the following theorem.

Theorem 1: The function $F(U, H)$ with $F_{U} \neq 0$ is a solution of Eq. (21) with polynomial dependence on $U$ if and only if it is of the form

$$
F(U, H)=P\left(\frac{U+\alpha H+\beta}{\gamma H+\delta}\right),
$$

where $P$ is a (nonconstant) polynomial and $\alpha, \beta, \gamma, \delta$ are constants with $|\gamma|^{2}+|\delta|^{2}>0$. 
Proof: Let

$$
F=a_{0}(H) U^{N}+a_{1}(H) U^{N-1}+\cdots+a_{N-1}(H) U+a_{N}(H)
$$

be a solution of (21) with $N \geqslant 1$ and $a_{0} \neq 0$. Substituting this expression into (21) and equating the coefficient of $U^{3 N-2}$ on both sides of the resulting expression, we find the condition $a_{0}^{\prime \prime} a_{0}=((N$ $+1) / N) a_{0}^{\prime 2}$, so $a_{0}(H)=(\gamma H+\delta)^{-N}$. Now we make the change of variables $\widetilde{U}=U /(\gamma H+\delta)$, $\widetilde{H}=\left(\phi H+{ }_{\rho}\right) /(\gamma H+\delta)$, where $\phi \delta-\gamma \rho \neq 0$. It follows that

$$
F=\widetilde{U}^{N}+\widetilde{a}_{1}(\widetilde{H}) \widetilde{U}^{N-1}+\cdots+\widetilde{a}_{N-1}(\widetilde{H}) \widetilde{U}+\widetilde{a}_{N}(\widetilde{H})
$$

in the new coordinates, and $F$ is a solution of

$$
F_{\tilde{H} \tilde{H}} F_{\tilde{U}}^{2}-2 F_{\tilde{U} \tilde{H}} F_{\tilde{U}} F_{\tilde{H}}+F_{\tilde{U} \tilde{U}} F_{\tilde{H}}^{2}=0 .
$$

Substituting the polynomial into (24) and equating coefficients of $\widetilde{U}^{3 N-3}$, we find $\widetilde{a}_{1}^{\prime \prime} N^{2}=0$ or $\widetilde{a}_{1}=\alpha_{1} \widetilde{H}+\beta_{1}$. Using this information, we return to our original expression for the polynomial and make a new change of variables of the form

$$
\widetilde{U}=\frac{U+\alpha H+\beta}{\gamma H+\delta}, \quad \widetilde{H}=\frac{\chi H+\zeta}{\gamma H+\delta},
$$

where $\chi \delta-\gamma \zeta \neq 0$, and $\alpha, \beta$ are chosen such that the transformed coefficient of $\widetilde{U}^{N-1}$ vanishes. In these variables

$$
F=\widetilde{U}^{N}+\widetilde{a}_{2}(\widetilde{H}) \widetilde{U}^{N-2}+\cdots+\widetilde{a}_{N-1}(\widetilde{H}) \widetilde{U}+\widetilde{a}_{N}(\widetilde{H})
$$

We substitute this expression into (24), and equating coefficients of $\widetilde{U}^{3 N-4}$ we find $\widetilde{a}_{2}^{\prime \prime}=0$, so $\widetilde{a}_{2}$ is a polynomial in $\widetilde{H}$ of order $\leqslant 1$. Proceeding in this fashion to equate coefficients of $\widetilde{U}^{3 N-s}$ for $s=5,6, \ldots$ in order, we find that the first occurence of $\widetilde{a}_{k}, k \geqslant 3$ in this sequence of equations takes the form $\widetilde{a}_{k}^{\prime \prime}=p_{k}\left(\widetilde{a}_{2}, \ldots, \widetilde{a}_{k-1}\right)$ where $p_{k}$ is a polynomial of order 3 at most. It follows by induction on $k$ that each $\tilde{a}_{k}$ is a polynomial in $\widetilde{H}$.

At this point we have shown that $F$ is a polynomial in both $\widetilde{U}$ and in $\widetilde{H}$. Let $\widetilde{H}^{M}$ be the maximal power of $\widetilde{H}$ that occurs in $F$. If $M=0$, we are done. Assume $M \geqslant 1$. If we use the argument of the first paragraph of this proof with $\widetilde{U}$ and $\widetilde{H}$ interchanged, we see that the coefficient of $\widetilde{H}^{M}$ in $F$ must take the form $\alpha_{0} /\left(\beta_{1} \widetilde{U}+1\right)$ with $\alpha_{0} \neq 0$. Since $F$ is a polynomial in $\widetilde{U}$ we must have $\beta_{1}=0$.

Thus

$$
F=\widetilde{U}^{N}+\widetilde{a}_{2}(\widetilde{H}) \widetilde{U}^{N-2}+\cdots+\widetilde{a}_{N-1}(\widetilde{H}) \widetilde{U}+\alpha_{0} \widetilde{H}^{M}
$$

Now substitute this expression into (24) and equate coefficients of $\widetilde{U}^{n} \widetilde{H}^{m}$ where $n+m$ is maximal. Suppose $N \geqslant M$. The highest power term in $F_{\tilde{H} \tilde{H}} F_{\tilde{U}}^{2}$ is $\alpha_{N} M(M-1) N^{2} \widetilde{H}^{M-2} \widetilde{U}^{2 N-2}$. The highest power term in $F_{\tilde{U}} \tilde{U}_{\tilde{H}}^{2}$ is $\alpha_{N}^{2} N(N-1) M^{2} \widetilde{H}^{2 M-2} \widetilde{U}^{N-2}$, but this is of lower order. The highest power term in $2 F_{\tilde{U} \tilde{H}} F_{\tilde{H}} F_{\tilde{U}}$ is $t=2 \alpha_{N} a_{N_{1}, M_{1}} N_{1} M_{1} N M \widetilde{U}^{N_{1}+N-2} \widetilde{H}^{M_{1}+M-2}$ where $a_{N_{1}, M_{1}}$ is the coefficient of $\widetilde{U}^{N_{1}} \widetilde{H}^{M_{1}}$ in $F$. Here $N_{1}<N, M_{1}<M$. If $N>N_{1}+M_{1}$, then the highest power term is the coefficient of $\widetilde{H}^{M-2} \widetilde{U}^{2 N-2}$, so $M=1$. If $N \leqslant N_{1}+M_{1}$, then $t=0$, so $a_{N_{1}, M_{1}}=0$. Thus, the only possiblity is $M=1$, so

$$
F=\widetilde{U}^{N}+\alpha_{2} \widetilde{U}^{N-2}+\cdots+\alpha_{N-1} \widetilde{U}+\alpha_{N} \widetilde{H}
$$


Substituting this expression into the differential equation we see that $F_{\tilde{U} \tilde{U}}=0$, or $F=\widetilde{U}+\alpha_{N} \widetilde{H}$. But this is impossible since $N=1$ and the coefficient of $\widetilde{U}^{N-1}$ must be 0 . Hence $F$ depends only on $\widetilde{U}$. There is a similar argument for the case $M>N$.

QED

If we limit our search for potentials to a space in which $U_{H}=f_{1}(x)$ is prescribed, then the general conditions (23) are replaced by

$$
F_{H}+f_{1}(F) F_{U}=0, \quad F_{U} \neq 0 .
$$

Equation (26) admits the complete integral

$$
F(U, H, \alpha, \beta)=f_{1}^{-1}\left(\frac{U+\alpha}{H+\beta}\right),
$$

where $f_{1}^{-1}$ is the function inverse to $f_{1}$. From this one can use standard techniques (method of characteristics, envelopes of solutions) from the theory of quasilinear first-order partial differential equations to construct solutions of (26) that satisfy particular initial conditions or that depend on arbitrary functions (Ref. 25, Chap. II or Ref. 26, Sec. 88).

Note: Standard Hamilton-Jacobi theory gives essentially these same constants of the motion, but from a different viewpoint. ${ }^{1}$ Our expression for $L_{3}$, for example, is

$$
L_{3}=\int U_{x}^{\prime-1} d p_{x}-\int U_{y}^{\prime-1} d p_{y}=M-N,
$$

where $U_{x}=-v_{1}(x)+f_{1}(x) H+L_{2}$, etc. Standard Hamilton-Jacobi theory gives

$$
L_{3}=\frac{1}{2} \int \frac{d x}{\sqrt{-v_{1}+f_{1} H+L_{2}}}-\frac{1}{2} \int \frac{d y}{\sqrt{-v_{2}+f_{2} H-L_{2}}}=\tilde{M}-\widetilde{N}
$$

In the standard theory $\widetilde{M}=\widetilde{M}\left(H, L_{2}, x\right)$, etc., whereas in our approach $M=M\left(H, L_{2}, p_{x}\right)$, etc. In both cases the condition (12) is satisfied. Our approach makes it easier in some cases to determine if polynomial invariants exist. It also points out the bracket relations between $M, N$ and the operators $L_{j}$ defining the separation, e.g., (11).

Examples abound of spaces for which these constructions apply. We illustrate this with a family of surfaces in Minkowski space: $d s^{2}=d z^{2}-d y^{2}-d x^{2}$. The surfaces involve a horispherical coordinate $\xi$ and take the form

$$
\mathbf{X}(t, \xi)=(x, y, z)=\left(2 t \xi, g(t)+\left(\xi^{2}-1\right) t, g(t)+\left(\xi^{2}+1\right) t\right) .
$$

The metric on the surface is

$$
d s^{2}=4\left[t g^{\prime}(t) d t^{2}-t^{2} d \xi^{2}\right]=4 t^{2}\left[d \rho^{2}-d \xi^{2}\right]=(f(\rho)+1)\left[d \rho^{2}-d \xi^{2}\right],
$$

where $(d \rho / d t)^{2}=g^{\prime}(t) / t^{2}$, and we can construct a polynomial invariant for the surface (and for an appropriate added potential) provided that the function $t^{2}=F(\rho)$ has a polynomial inverse function, i.e., $\rho=G\left(t^{2}\right)$ where $G$ is a polynomial. Clearly $g^{\prime}(t)=4 t^{4} G^{\prime}\left(t^{2}\right)^{2}$ and any polynomial $G$ will determine a surface with a polynomial invariant. For example, choose $G\left(t^{2}\right)=\frac{1}{2} t^{4}+t^{2}$. Then we can take $g(t)=\frac{4}{9} t^{9}+\frac{8}{7} t^{7}+\frac{4}{5} t^{5}$ and $\rho(t)=\frac{1}{2} t^{4}+t^{2}$. The resulting $M$ will be third-order polynomial in $p_{\xi}$ and $p_{\rho}$. Similarly, we can determine a potential term $v(\rho)$ with $v^{\prime} \neq 0$ such that $\mathrm{N}$ is a polynomial in $p_{\xi}$ and $p_{\rho}$.

Rather than make either of the choices $p_{x}$ or $x$ for the independent variable in (12) we could choose some other function $w\left(x, p_{x}\right)$, adapted to the specific problem at hand. For example, let us take $w\left(x, p_{x}\right)=r^{\prime}(x) p_{x}$ for some given function $r$, and require $M=M\left(H, L_{2}, w\right)$. Solving (12) in these variables we find 


$$
M=\int \frac{d r(x)}{d w^{2}} d w
$$

where

$$
w^{2}=U=r^{\prime}(x)^{2} p_{x}^{2}=r^{\prime}(x)^{2}\left(-v_{1}+f_{1} H+L_{2}\right), \quad r(x)=F\left(U, H, L_{2}\right) .
$$

This approach will work even if $v_{1}$ and $f_{1}$ are constants; it is guaranteed to yield a polynomial invariant if we require

$$
r=P_{n}\left(\frac{U+\alpha_{1} H+\alpha_{2} L_{2}+\alpha_{3}}{\alpha_{4} H+\alpha_{5} L_{2}+\alpha_{6}}\right),
$$

where $P_{n}$ is a polynomial of order $n$ and the $\alpha_{i}$ are constants. Then there exists a function $F_{n}$, inverse to $P_{n}$, such that

$$
U=\left(\alpha_{4} H+\alpha_{5} L_{2}+\alpha_{6}\right) F_{n}(r)-\left(\alpha_{1} H+\alpha_{2} L_{2}+\alpha_{3}\right)=r^{\prime 2}\left(-v_{1}+f_{1} H+L_{2}\right) .
$$

Equating coefficients of $L_{2}$ we find the condition $r^{\prime}(x)^{2}=\alpha_{5} F_{n}(r)-\alpha_{2}$ and we can solve for $r(x)$ by quadratures. Equating coefficients of $H$ and the constant term, we obtain expressions for $f_{1}$ and $v_{1}$ :

$$
f_{1}(x)=\frac{\alpha_{4} F_{n}(r)-\alpha_{1}}{\alpha_{5} F_{n}(r)-\alpha_{2}}, \quad v_{1}(x)=\frac{\alpha_{3}-\alpha_{6} F_{n}(r)}{\alpha_{5} F_{n}(r)-\alpha_{2}}
$$

It follows that $\left(\alpha_{4} H+\alpha_{5} L_{2}+\alpha_{6}\right)^{n} M\left(r p_{x}\right)$ is a polynomial in the momenta.

\section{LIE FORM AND NONORTHOGONAL SEPARATION IN TWO DIMENSIONS}

We know that if a Hamiltonian

$$
H=\sum_{i, j=1}^{2} g^{i j} p_{i} p_{j}
$$

admits a constant of the motion $L$ that is quadratic in the momenta

$$
L=\sum_{i, j=1}^{2} a^{i j} p_{i} p_{j}, \quad\{H, L\}=0
$$

and if the roots of the determinant $\left|a^{i j}-\lambda g^{i j}\right|$ are distinct, then the eigenforms define new (separable) variables $\rho, \mu$ and the Hamiltonian can be written in Liouville form

$$
H=\frac{p_{\rho}^{2}+p_{\mu}^{2}}{f(\rho)+g(\mu)} .
$$

However, it may be that the roots of this determinant are equal. In this case $H$ cannot be put into Liouville form, but rather Lie form, which for a suitable choice of variables (nonseparable) is

$$
H=\frac{p_{x} p_{y}}{x+B(y)}
$$

The associated quadratic constant of the motion is

$$
L=p_{x}^{2}-2 y H
$$


We now ask the question: When the roots of $L$ are equal, how can we calculate the third invariant? We are interested in the the same question when a potential is added to the Hamiltonian. These questions can readily be answered. Indeed if we look for a function $N\left(H, L, y, p_{y}\right)$ that is in involution with $H$, we obtain the equation

$$
(x+B(y)) N_{y}+p_{y} B^{\prime}(y) N_{p_{y}}=0 .
$$

If we solve (31) and (32) for $x$ and $p_{x}$ in terms of the variables $H, L, y$ and $p_{y}$, we obtain

$$
p_{x}=\sqrt{L+2 y H}, \quad x=\frac{p_{y}}{H} \sqrt{L+2 y H}-B(y) .
$$

The equation (33) for $N$ then has the form

$$
\frac{\sqrt{L+2 y H}}{H B^{\prime}(y)} N_{y}+N_{p_{y}}=0 .
$$

From this condition a second invariant can be readily obtained in the form

$$
L^{\prime}=H \int \frac{B^{\prime}(y)}{\sqrt{L+2 y H}} d y-p_{y} .
$$

We now extend these considerations by considering the possibility of adding a potential. If we do this and have an extra quadratic constant, then $H$ and $L$ have the forms

$$
H=\frac{p_{x} p_{y}+\frac{1}{2} K(y)}{x+B(y)}+\frac{1}{2} U^{\prime}(y), \quad L=p_{x}^{2}-2 y H+U(y) .
$$

Solving (35) for $p_{x}$ and $x$ gives

$$
p_{x}=\sqrt{L-U(y)+2 y H}, \quad x=\frac{p_{y} \sqrt{L-U(y)+2 y H}+\frac{1}{2} K(y)}{H-\frac{1}{2} U^{\prime}(y)}-B(y) .
$$

Then the equation for $N$ has the form

$$
\begin{array}{r}
2 \sqrt{L-U(y)+2 y H}\left(2 H-U^{\prime}(y)\right) N_{y}+\left[-2 U^{\prime \prime}(y) \sqrt{L-U(y)+2 y H} p_{y}+B^{\prime}(y) U^{\prime}(y)^{2}\right. \\
\left.+4 B^{\prime}(y) H^{2}-U^{\prime \prime}(y) K(y)-4 B^{\prime}(y) U^{\prime}(y) H+K^{\prime}(y) U^{\prime}(y)-2 K^{\prime}(y) H\right] N_{p_{y}}=0 .
\end{array}
$$

This equation can, in principle, be solved directly. In fact, for suitable redefinition of the variables $y \rightarrow Y, p_{y} \rightarrow P_{y}$, Eq. (36) can be put in the form

$$
N_{Y}+\left(P_{Y}+s(Y)\right) N_{P_{Y}}=0
$$

that can be solved by the further transformation

$$
P_{Y^{\prime}}=P_{Y}+t(Y), \quad Y^{\prime}=Y .
$$

Then, provided that

$$
t^{\prime}(Y)-t(Y)+s(Y)=0
$$

(37) reduces to 


$$
N_{Y^{\prime}}+P_{Y^{\prime}} N_{P_{Y^{\prime}}}=0
$$

From this we immediately deduce an extra constant of the motion of the form

$$
L^{\prime}=e^{Y^{\prime}} / P_{Y^{\prime}} .
$$

The equation for $t(Y)$ has the solution

$$
t(Y)=e^{Y} \int^{Y} e^{-u} s(u) d u .
$$

There is one remaining possibility for a quadratic constant of the motion (30) in two dimensions: the constant may be associated with nonorthogonal separation of variables. In two dimensions there is only one case: separation in light cone (null) coordinates. ${ }^{27}$ For this case the Hamiltonian takes the form

$$
H=p_{z} p_{\bar{z}}+f(\bar{z}),
$$

and there is a Killing vector $p_{z}$, so $p_{z}^{2}$ is a second-order constant of the motion. In addition there is a quadratic constant

$$
L=M p_{z}+\frac{i}{2} \int \bar{z} \frac{d f}{d \bar{z}} d \bar{z}
$$

Thus we have answered the following questions.

(1) If a Hamiltonian with potential admits a quadratic constant of the motion in two dimensions how does one calculate the third constant?

(2) A subset of problem 1 is when we require separation only and ask to calculate the third constant.

\section{SYSTEMS IN THREE DIMENSIONS}

Let us now look at how the orthogonal separation of variable considerations extend to three dimensions. If we have a general separable coordinate system in three dimensions, we could take the Hamiltonian to be $\mathrm{b}^{20,28,29}$

$$
H=L_{1}=\frac{g_{2}-g_{3}}{\Phi}\left(p_{x_{1}}^{2}+v_{1}\left(x_{1}\right)\right)+\frac{g_{3}-g_{1}}{\Phi}\left(p_{x_{2}}^{2}+v_{2}\left(x_{2}\right)\right)+\frac{g_{1}-g_{2}}{\Phi}\left(p_{x_{3}}^{2}+v_{3}\left(x_{3}\right)\right),
$$

where $g_{i}=g_{i}\left(x_{i}\right), f_{i}=f\left(x_{i}\right)$ and $\Phi$ is the determinant of the Stäckel matrix

$$
\left(\begin{array}{lll}
1 & f_{1} & g_{1} \\
1 & f_{2} & g_{2} \\
1 & f_{3} & g_{3}
\end{array}\right) .
$$

This automatically gives us two more invariants:

$$
\begin{gathered}
L_{2}=\frac{f_{3}-f_{2}}{\Phi}\left(p_{x_{1}}^{2}+v_{1}\left(x_{1}\right)\right)+\frac{f_{1}-f_{3}}{\Phi}\left(p_{x_{2}}^{2}+v_{2}\left(x_{2}\right)\right)+\frac{f_{2}-f_{1}}{\Phi}\left(p_{x_{3}}^{2}+v_{3}\left(x_{3}\right)\right), \\
L_{3}=\frac{f_{2} g_{3}-f_{3} g_{2}}{\Phi}\left(p_{x_{1}}^{2}+v_{1}\left(x_{1}\right)\right)+\frac{f_{3} g_{1}-f_{1} g_{3}}{\Phi}\left(p_{x_{2}}^{2}+v_{2}\left(x_{2}\right)\right)+\frac{f_{1} g_{2}-f_{2} g_{1}}{\Phi}\left(p_{x_{3}}^{2}+v_{3}\left(x_{3}\right)\right) .
\end{gathered}
$$


We need to find an additional two invariants, such that the five form a functionally independent set.

If we look for a function $M_{1}$ such that

$$
\left\{H, M_{1}\right\}=\frac{g_{2}-g_{3}}{\Phi},
$$

then this function satisfies the equation

$$
2 p_{x_{1}} \partial_{x_{1}} M_{1}+\left[-v_{1}^{\prime}\left(x_{1}\right)+f_{1}^{\prime} H+g_{1}^{\prime} L_{2}\right] \partial_{p_{x_{1}}} M_{1}=1
$$

which looks like the form we have been using in two dimensions. There are similar equations for the corresponding functions $M_{i}$ for $i=2,3$. For $M_{1}\left(H, L_{2}, L_{3}, Q_{1}\right)$ with $Q_{1}=p_{x_{1}}$ this has the solution

$$
M_{1}=\int U_{1}^{\prime-1} d Q_{1}
$$

where $U_{1}\left(x_{1}\right)=-v_{1}\left(x_{1}\right)+f_{1} H+g_{1} L_{2}+L_{3}$ and $L_{3}=v_{1}-f_{1} H-g_{1} L_{2}+p_{x_{1}}^{2}$. (Here, we consider $U_{1}^{\prime-1}=d x_{1} / d U_{1}$ to be a function of $U_{1}=Q_{1}^{2}$ to compute the integral. We also assume that $\left|v_{1}^{\prime}\right|$ $+\left|f_{1}^{\prime}\right|+\left|g_{1}^{\prime}\right|>0$.) The corresponding invariant that we can calculate from these three functions is $L_{3}^{\prime}=M_{1}+M_{2}+M_{3}$. This is based on the obvious identity

$$
\left(g_{2}-g_{3}\right)+\left(g_{3}-g_{1}\right)+\left(g_{1}-g_{2}\right)=0 .
$$

Note: As in the two dimensional case, the solution of the equation (44) can be understood in a more general context. We have the dual relations

$$
x=F\left(U-L_{3}, H, L_{2}\right), \quad U\left(x, H, L_{2}\right)=-v_{1}(x)+f_{1}(x) H+g_{1}(x) L_{2}+L_{3},
$$

where $U_{x} \neq 0$. (Since $U$ and $L_{3}$ occur only as $U-L_{3}$ we can, without loss of generality, set $L_{3}$ $=0$ in the equations immediately following, and then replace $U$ by $U-L_{3}$ in the examples.) Thus we have

$$
1=F_{U} U_{x}, \quad F_{U} U_{H}+F_{H}=0, \quad F_{U} U_{L_{2}}+F_{L_{2}}=0 .
$$

The condition that $U\left(x, H, L_{2}\right)$ is linear in $H$ and $L_{2}$, i.e., $U_{H H}=U_{L_{2} L_{2}}=U_{H L_{2}}=0$, leads to the following necessary and sufficient conditions that the function $x=F\left(U, H, L_{2}\right)$ correspond to an invariant $M_{1}$ on a Riemannian manifold with potential:

$$
\begin{gathered}
F_{H H} F_{U}^{2}-2 F_{U H} F_{U} F_{H}+F_{U U} F_{H}^{2}=0, \quad F_{U} \neq 0, \\
F_{U U} F_{L_{2}}^{2}-2 F_{L_{2} U} F_{L_{2}} F_{U}+F_{L_{2} L_{2}} F_{U}^{2}=0, \\
F_{L_{2} L_{2}} F_{H}^{2}-2 F_{H L_{2}} F_{H} F_{L_{2}}+F_{H H} F_{L_{2}}^{2}=0 .
\end{gathered}
$$

These equations admit an infinite dimensional conformal symmetry group. Indeed, if $V$ $=F\left(U, H, L_{2}\right)$ is a solution, then $G(V)$ is also a solution, for any nonconstant function $G$. Also, this group contains the subgroup of inhomogeneous affine symmetries: if $F\left(U, H, L_{2}\right)$ is a solution, then so is $F\left(\left[a_{11} U+a_{12} H+a_{13} L_{2}+a_{14}\right] / A,\left[a_{21} U+a_{22} H+a_{23} L_{2}+a_{24}\right] / A,\left[a_{31} U+a_{32} H\right.\right.$ $\left.\left.+a_{33} L_{2}+a_{24}\right] / A\right)$ where $a_{i j}$ are constants, $\operatorname{det}\left(a_{i j}\right) \neq 0$ and

$$
A=a_{41} U+a_{42} H+a_{43} L_{2}+a_{44} .
$$



form.

As in the two dimensional case, the only polynomial functions $F$ of $U$ are of a very special

Theorem 2: The function $F\left(U, H, L_{2}\right)$ with $F_{U} \neq 0$ is a solution of Eqs. (46) with polynomial dependence on $U$ if and only if it is of the form

$$
F\left(U, H, L_{2}\right)=P\left(\frac{U+\alpha_{1} H+\alpha_{2} L_{2}+\beta}{\gamma_{1} H+\gamma_{2} L_{2}+\delta}\right),
$$

where $P$ is a (nonconstant) polynomial and $\alpha_{i}, \beta, \gamma_{i}, \delta$ are constants with $\left|\gamma_{1}\right|^{2}+\left|\gamma_{2}\right|^{2}+|\delta|^{2}$ $>0$.

Proof: The proof is similar to that of Theorem 1. It follows from this theorem and the first two equations in (46) that

$$
F=P^{(1)}\left(U^{(1)}, L_{2}\right)=P^{(2)}\left(U^{(2)}, H\right),
$$

where the $P^{(i)}$ are polynomials of strict order $N$ in their first arguments and

$$
U^{(1)}=\frac{U+\alpha_{1}^{(1)} L_{2} H+\beta^{(1)} L_{2}}{\gamma_{1}^{(1)} L_{2} H+\delta^{(1)} L_{2}} \quad U^{(2)}=\frac{U+\alpha_{1}^{(2)} H L_{2}+\beta^{(2)} H}{\gamma_{1}^{(2)} H L_{2}+\delta^{(2)} H} .
$$

Furthermore, the coefficients of the $N-1$-st power of their first arguments can be asumed to be zero. Comparing the coefficients of the highest power $U^{N}$ of $U$ in $F$, we see that this coefficient must be of the form

$$
\left(\gamma_{1} H+\gamma_{2} L_{2}+\gamma_{3} H L_{2}+\delta\right)^{-N},
$$

where now the $\gamma_{i}, \delta$ are constants. Substituting this into the third equation in (46) and equating coefficients of $U^{3 N}$, we see that $\gamma_{3}=0$.

Equating the coefficients of $U^{N-1}$ in the $P^{(i)}$ we see that

$$
U^{(1)}=U^{(2)}=\widetilde{U}=\frac{U+\alpha_{1} H+\alpha_{2} L_{2}+\phi H L_{2}+\beta}{\gamma_{1} H+\gamma_{2} L_{2}+\delta},
$$

where the coefficients are constants. Then, substituting this result into the third equation again and comparing coefficients of $U^{3 N-1}$ we see that $\phi=0$. At this point we have shown that $F$ $=P\left(\widetilde{U}, H, L_{2}\right)$ where $P$ is a polynomial of order exactly $N$ in its first argument. The proof that $P$ is independent of its second and third arguments follows exactly as in the last part of the proof of Theorem 1.

QED

If we limit our search for potentials to a space in which $U_{H}=f_{1}(x), U_{L_{2}}=g_{1}(x)$ are prescribed, then the general conditions (46) are replaced by

$$
F_{H}+f_{1}(F) F_{U}=0, \quad F_{L_{2}}+g_{1}(F) F_{U}=0, \quad F_{U} \neq 0 .
$$

From this one can use standard techniques (method of characteristics, envelopes of solutions) from the theory of systems of quasilinear first order partial differential equations to construct solutions of (47) that satisfy particular initial conditions or that depend on arbitrary functions.

The invariant $L_{3}^{\prime}=M_{1}+M_{2}+M_{3}$ also commutes with $L_{2}$. Indeed, from the fact that

$$
\partial_{x_{1}} L_{2}=\frac{f_{3}-f_{2}}{\Phi}\left(v_{1}^{\prime}-f_{1}^{\prime} H-g_{1}^{\prime} L_{2}\right)
$$

we can verify that (44) implies 


$$
\left\{L_{2}, M_{1}\right\}=\frac{f_{3}-f_{2}}{\Phi}
$$

The corresponding conditions are satisfied by $M_{2}$ and $M_{3}$. Then the fact that $\left\{L_{2}, L_{3}^{\prime}\right\}=0$ is implied by the obvious identity

$$
\left(f_{3}-f_{2}\right)+\left(f_{1}-f_{3}\right)+\left(f_{2}-f_{1}\right)=0 .
$$

Finally, from the fact that

$$
\partial_{x_{1}} L_{3}=\frac{f_{2} g_{3}-f_{3} g_{2}}{\Phi}\left(v_{1}^{\prime}-f_{1}^{\prime} H-g_{1}^{\prime} L_{2}\right)
$$

we can verify that (44) implies

$$
\left\{L_{3}, M_{1}\right\}=\frac{f_{2} g_{3}-f_{3} g_{2}}{\Phi} .
$$

The corresponding conditions are satisfied by $M_{2}$ and $M_{3}$. Then the fact that $\left\{L_{3}, L_{3}^{\prime}\right\}=1$ is implied by the identity

$$
\left(f_{2} g_{3}-f_{3} g_{2}\right)+\left(f_{3} g_{1}-f_{1} g_{3}\right)+\left(f_{1} g_{2}-f_{2} g_{1}\right)=\Phi .
$$

Similarly, we can define a new invariant $L_{2}^{\prime}$ by requiring that a new function $M_{1}$ satisfy

$$
\left\{L_{1}, M_{1}\right\}=\frac{g_{1}\left(g_{2}-g_{3}\right)}{\Phi},
$$

with analogous conditions for $M_{2}$ and $M_{3}$. For $M_{1}\left(H, L_{2}, L_{3}, Q_{1}\right)$ with $Q_{1}=p_{x_{1}}$ this has the solution

$$
M_{1}=\int g_{1} U_{1}^{\prime-1} d Q_{1}
$$

where $U_{1}\left(x_{1}\right)=-v_{1}\left(x_{1}\right)+f_{1} H+g_{1} L_{2}+L_{3}$.

[Note that for $M_{1}$ to be a polynomial in $p_{x}, p_{y}, p_{z}$ we must have $g_{1}(F) F_{U}$ a polynomial in $U$. If $g_{1}^{\prime}=0$, this reduces to requiring $F$ to be a polynomial in $U$. If $g_{1}^{\prime} \neq 0$, we can replace the variable $x$ by $\widetilde{x}_{1}=r\left(x_{1}\right)=\int g_{1}\left(x_{1}\right) d x_{1}$ with $\widetilde{x}_{1}=G\left(U, H, L_{2}, L_{3}\right)$. Then $g_{1}(F) F_{U}=G_{U}$ and our original analysis goes through with $F$ replaced by $G$. It is guaranteed to yield a polynomial invariant if we require

$$
r=P_{n}\left(\frac{U+\alpha_{1} H+\alpha_{2} L_{2}+\alpha_{3} L_{3}+\alpha_{4}}{\alpha_{5} H+\alpha_{6} L_{2}+\alpha_{7}}\right), \quad g_{1}=r^{\prime}\left(x_{1}\right),
$$

where $P_{n}$ is a polynomial of order $n$ and the $\alpha_{i}$ are constants. Then there exists a function $F_{n}$, inverse to $P_{n}$, such that

$$
U=\left(\alpha_{5} H+\alpha_{6} L_{2}+\alpha_{7}\right) F_{n}(r)-\left(\alpha_{1} H+\alpha_{2} L_{2}+\alpha_{3} L_{3}+\alpha_{4}\right)=-v_{1}+f_{1} H+g_{1} L_{2}+L_{3} .
$$

Equating coefficients of $L_{2}$ we find the condition $r^{\prime}=\alpha_{6} F_{n}(r)-\alpha_{2}$ and we can solve for $r\left(x_{1}\right)$ by quadratures. Equating coefficients of $H, L_{3}$ and the constant term, we find $\alpha_{3}=-1$ and

$$
f_{1}(x)=\alpha_{5} F_{n}(r)-\alpha_{1}, \quad g_{1}(x)=\alpha_{6} F_{n}(r)-\alpha_{2}, \quad v_{1}(x)=\alpha_{4}-\alpha_{7} F_{n}(r) .
$$

It follows that $\left(\alpha_{5} H+\alpha_{6} L_{2}+\alpha_{7}\right)^{n} M_{1}$ is a polynomial in the momenta.] 
The corresponding invariant that we can calculate from these three functions is $L_{2}^{\prime}=M_{1}$ $+M_{2}+M_{3}$. This is based on the obvious identity

$$
g_{1}\left(g_{2}-g_{3}\right)+g_{2}\left(g_{3}-g_{1}\right)+g_{3}\left(g_{1}-g_{2}\right)=0 \text {. }
$$

Then it follows that

$$
\left\{L_{2}, M_{1}\right\}=\frac{g_{1}\left(f_{3}-f_{2}\right)}{\Phi}, \quad\left\{L_{3}, M_{1}\right\}=\frac{g_{1}\left(f_{2} g_{3}-f_{3} g_{2}\right)}{\Phi},
$$

with analogous results for $M_{2}, M_{3}$. Thus, from the definition of $\Phi$ we see that $\left\{L_{2}, L_{2}^{\prime}\right\}=1$.

Finally, we define a function $L_{1}^{\prime}=M_{1}+M_{2}+M_{3}$ by requiring

$$
\left\{L_{1}, M_{1}\right\}=\frac{f_{1}\left(g_{2}-g_{3}\right)}{\Phi}
$$

with similar conditions for $M_{2}$ and $M_{3}$. For $M_{1}\left(H, L_{2}, L_{3}, Q_{1}\right)$ with $Q_{1}=p_{x_{1}}$ this has the solution

$$
M_{1}=\int f_{1} U_{1}^{\prime-1} d Q_{1}
$$

Then it follows that

$$
\left\{L_{2}, M_{1}\right\}=\frac{f_{1}\left(f_{3}-f_{2}\right)}{\Phi}, \quad\left\{L_{3}, M_{1}\right\}=\frac{f_{1}\left(f_{2} g_{3}-f_{3} g_{2}\right)}{\Phi}
$$

with analogous relations for $M_{2}$ and $M_{3}$.

In summary, all brackets between the six functions $L_{i}, L_{i}^{\prime}$ are zero except that

$$
\left\{L_{3}, L_{3}^{\prime}\right\}=\left\{L_{2}, L_{2}^{\prime}\right\}=\left\{L_{1}, L_{1}^{\prime}\right\}=1 \text {. }
$$

Thus the mapping $\left(x_{1}, x_{2}, x_{3}, p_{x_{1}}, p_{x_{2}}, p_{x_{3}}\right) \rightarrow\left(L_{1}, L_{2}, L_{3}, L_{1}^{\prime}, L_{2}^{\prime}, L_{3}^{\prime}\right)$ is canonical.

Note: Standard Hamilton-Jacobi theory gives exactly these same constants of the motion, from a different viewpoint. ${ }^{1}$ Our expression for $L_{3}^{\prime}$, for example, is

$$
L_{3}^{\prime}=\sum_{j} \int U_{j}^{\prime-1} d p_{x_{j}}=\sum_{j} M_{j}
$$

where $U_{j}=-v_{j}\left(x_{j}\right)+f_{j} L_{1}+g_{j} L_{2}+L_{3}$ and $U_{j}=p_{x_{j}}^{2}$. Standard Hamilton-Jacobi theory gives

$$
L_{3}^{\prime}=\frac{1}{2} \sum_{j} \int \frac{d x_{j}}{\sqrt{-v_{j}+f_{j} L_{1}+g_{j} L_{2}+L_{3}}}=\sum_{j} \tilde{M}_{j}
$$

In the standard theory $\tilde{M}_{j}=\tilde{M}_{j}\left(L_{1}, L_{2}, L_{3}, x_{j}\right)$, whereas in our approach $M_{j}$ $=M_{j}\left(L_{1}, L_{2}, L_{3}, p_{x_{j}}\right)$. In both cases the condition (44) is satisfied. Our approach makes it straightforward to determine exactly when the $L_{i}^{\prime}$ are polynomials in the momenta $p_{x_{j}}$. It also points out the bracket relations between the $M_{i}$ and the operators $L_{j}$ defining the separation, e.g., (43), (48), (49), (51), and (53).

The generalization to $n$ dimensions is straightforward.

${ }^{1}$ V. I. Arnold, "Mathematical Methods of Classical Mechanics," (translated by K. Vogtmann and A. Weinstein), Graduate Texts in Mathematics, Vol. 60, (Springer-Verlag, New York, 1978).

${ }^{2}$ M. Karlovini and K. Rosquist, Gen. Relativ. Gravit. 31, 1271 (1999).

${ }^{3}$ M. A. Ushveridze, J. Phys. A 21, 1601 (1988). 
${ }^{4}$ N. W. Evans, Phys. Rev. A 41, 5666 (1990).

${ }^{5}$ N. W. Evans, J. Math. Phys. 32, 3369 (1991).

${ }^{6}$ N. W. Evans, Phys. Lett. A 147, 483 (1990).

${ }^{7}$ S. Wojciechowski, Phys. Lett. A 95, 279 (1983).

${ }^{8}$ L. P. Eisenhart, Phys. Rev. 74, 87 (1948).

${ }^{9}$ J. Friš, V. Mandrosov, Ya. A. Smorodinsky, M. Uhlir, and P. Winternitz, Phys. Lett. 16, 354 (1965).

${ }^{10}$ J. Frišs, Ya. A. Smorodinskii, M. Uhlír, and P. Winternitz, Sov. J. Nucl. Phys. 4, 444 (1967).

${ }^{11}$ A. A. Makarov, Ya. A. Smorodinsky, Kh. Valiev, and P. Winternitz, Nuovo Cimento A 52, 1061 (1967).

${ }^{12}$ D. Bonatos, C. Daskaloyannis, and K. Kokkotas, Phys. Rev. A 50, 3700 (1994).

${ }^{13}$ F. Calogero, J. Math. Phys. 10, 2191 (1969).

${ }^{14}$ A. Cisneros and H. V. McIntosh, J. Math. Phys. 10, 277 (1969).

${ }^{15}$ L. G. Mardoyan, G. S. Pogosyan, A. N. Sissakian, and V. M. Ter-Antonyan, Nuovo Cimento Soc. Ital. Fis., B 88, 43 (1985); Theor. Math. Phys. 61, 1021 (1984); J. Phys. A 18, 455 (1985).

${ }^{16}$ B. Zaslow and M. E. Zandler, Am. J. Phys. 35, 1118 (1967).

${ }^{17}$ J. Hietarinta, Phys. Rep. 147, 87 (1987).

${ }^{18}$ M. F. Ranada and M. Santander, Phys. Lett. A 278, 271 (2001).

${ }^{19}$ P. Stäckel, Hàbilitationsschrift,Universität Halle, 1891. [See Jahrbuch uber die Fortschritte der Mathematik 23, 402 (1891).]

${ }^{20}$ L. P. Eisenhart, Ann. Math. 35(2), 284 (1934).

${ }^{21}$ E. G. Kalnins, J. Kress, W. Miller, Jr., and G. S. Pogosyan, J. Phys. A 34, 4705 (2001).

${ }^{22}$ W. Miller, Jr., Symmetry and Separation of Variables (Addison-Wesley, Providence, RI, 1977).

${ }^{23}$ E. G. Kalnins, W. Miller, Jr., and G. S. Pogosyan, J. Math. Phys. 37, 6439 (1996).

${ }^{24}$ J. A. Sethian, "Level Set Methods and Fast Marching Methods Evolving Interfaces," in Computational Geometry, Fluid Mechanics, Computer Vision, and Materials Science, Cambridge Monograph on Applied and Computational Mathematics (Cambridge University Press, Cambridge, 1999), Chap. 1.

${ }^{25}$ R. Courant and D. Hilbert, Methods of Mathematical Physics, Vol. II (Interscience, New York, 1962).

${ }^{26}$ D. Zwillinger, Handbook of Differential Equations (Academic, San Diego, CA, 1989).

${ }^{27}$ E. G. Kalnins, Separation of Variables for Riemannian Spaces of Constant Curvature, Pitman Monographs and Surveys in Pure and Applied Mathematics 28 (Longman, Essex, U.K., 1986).

${ }^{28}$ E. G. Kalnins and W. Miller, J. Diff. Geom. 14, 221 (1979).

${ }^{29}$ E. G. Kalnins and W. Miller, SIAM (Soc. Ind. Appl. Math.) J. Math. Anal. 11, 1011 (1980). 